\title{
Injectable scaffolds for bone regeneration
}

Sabina Yasmeen, Man Kit Lo, Salina Bajracharya and Marta Roldo*

School of Pharmacy and Biomedical Science, University of Portsmouth, St Michael's Building, White Swan Road, Portsmouth, UK. Fax:0044 2392843565; Tel:0044 2392843586; email:marta.roldo@port.ac.uk

Keywords: thermosensitive gels; chitosan; carbon nanotubes; hydroxyapatite; bone regeneration

Materials abbreviations: CS, chitosan; HA, hydroxyapatite; HACS, hydroxyapatatite/chitosan composite; CNTs, carbon nanotubes; SWNTs, single-wall carbon nanotubes; MWNTs, multiwall carbon nanotubes; CS-HA, chitosan gel containing hydroxyapatite; CS-HACS, chitosan gel containing hydroxyapatite/chitosan composite; CS-HACS-CNTs, chitosan gel containing hydroxyapatite/chitosan composite and chitosan-grafted carbon nanotubes (either SWNTs or MWNTs).

$\S$ Supporting information

\section{ABSTRACT}

Clinical treatments of significant bone defects involve invasive procedures such as the application of auto- and allo-grafts. These procedures present many limitations including the potential for infection and rejection. There is therefore a need to develop novel therapeutic strategies able to exploit the natural regenerative potential of bone and that can be delivered in a 
less invasive manner. Amongst the materials studied for the development of novel scaffolds, stimuli-responsive gels containing hydroxyapatite and carbon nanotubes as nanofillers have generated great interest. In the present work, chitosan gels containing chitosan grafted CNTs and chitosan-hydroxyapatite complex have been formed by crosslinking with glycerol phosphate. The addition of the nanofillers afforded hydrogels with a faster sol/gel transition at $37^{\circ} \mathrm{C}$ and enhanced mechanical properties. The thermosensitive composite gels also showed a good bioactivity profile associated with potential for the prolonged delivery of protein drugs. The inclusion of chemically crosslinked CNTs and HA in thermosensitive gels afforded injectable composite materials with enhanced properties, including reduction of gelation time, improved mechanical properties, good bioactivity and prolonged drug release.

\section{Introduction}

Standard clinical treatments of significant bone defects involve the application of auto- and allo- grafts which present serious limitations and constitute an invasive procedure ${ }^{1,2}$. Novel therapeutic strategies aim at exploiting the natural regenerative potential of bone by providing biocompatible scaffolds that sustain cell migration, division and proliferation. Amongst the materials studied for the development of novel scaffolds, hydrogels are very prominent ${ }^{3}$. Their biocompatibility and ability to provide controlled release of drugs and growth factors are however counteracted by their weak mechanical properties and low bioactivity. Recently, the addition of nanofillers has been investigated as a viable strategy to address these downfalls. Carbon nanotubes have been proposed for their ability to increase the mechanical strength of hydrogels and hydroxyapatite (HA) for its bioactive role ${ }^{4}$. Furthermore, the use of thermosensitive hydrogels could overcome the limitations of invasive procedures, substituting solid implants with materials that gel in situ after injection. 
Different approaches for the production of solid composite scaffolds have been reported in literature. Fonseca-Garcia et al. prepared chitosan-HA-MWNTs composite scaffolds by a simple method that involved the freeze drying of the physical mixture of the three components in aqueous suspension. They obtained 3D macroporous biomimetic scaffolds with good biocompatibility and potential as support materials for bone tissue engineering. Furthermore, they looked at the effect of CNTs doping on cell viability and found that non-doped and oxygendoped MWNTs afforded materials with higher biocompatibility compared to nitrogen-doped tubes $^{5}$. A similar procedure was used by Im et al., who investigated the effect of magnetically synthesised SWNTs on the biocompatibility of the composite scaffolds. They demonstrated that the narrower and longer magnetically synthesised CNTs had a positive effect on osteoblast attachment, furthermore the mechanical properties of these materials were also enhanced ${ }^{6}$. Better material integration can be obtained by co-synthesising the individual components of the composite scaffolds. Porous HA/CNTs scaffolds were prepared by Lu et al. by a two-step process that involved the synthesis of HA in the presence of a water soluble polymer to favour formation of pores and the successive synthesis of CNTs on the scaffolds by chemical vapour deposition. The addition of CNTs to these structures enhanced the mechanical properties and afforded a superparamagnetic material that can favour attraction of bioactive molecules and cells involved in bone regeneration ${ }^{7}$. Conversely, Chen et al. synthesised HA in the presence of preformed CNTs and chitosan and then added a crosslinker to form a hydrogel. CNTs enhanced the elastic modulus and compressive strength of the gel in a concentration dependent manner, the new composites also showed good cell adherence and spreading ${ }^{2}$. All of the materials described above provide solid scaffolds that require surgery in order to be placed on the required site, a way to overcome this limitation is the development of stimuli-responsive systems that gel in situ. 
Tang et al. evaluated the effects of the addition of HA to thermosensitive chitosan/PVA hydrogels. Both ex situ and in situ strategies have been used for the introduction of the HA into the gels, with the latter method affording a material with better chemical interactions between its components and a better performance both in terms of mechanical properties and of control over drug release rate ${ }^{8}$. The disadvantage of this system is however that the drug, BSA in this study, was added simultaneously to HA precursors so this may lead to instability, above all when more sensitive protein drugs, such as growth factors, are loaded. Gao et al. also developed HA loaded thermosensitive chitosan gels, in this case the drug was loaded onto preformed HA nanoparticles. They did not study the release profile of the encapsulated drugs but a positive effect on the proliferation of mesenchymal stem cells was reported ${ }^{9}$.

In the present study we aim at developing a thermosensitive system with enhanced interaction between the three constituent components. The gel is based on electrostatically crosslinked chitosan $^{10}$, the integration with HA and CNTs is favoured by introduction of grafted chitosan on the surface of carboxylated CNTs and by wet precipitation of HA in the presence of chitosan, in this way all three components will interact with the crosslinker, glycerol phosphate. The obtained gels underwent physico-chemical characterisation and their ability to provide a platform for the controlled release of protein drugs is evaluated.

2. Materials and methods

\subsection{Materials}

Functionalised MWNTs (carboxylation $2.56 \mathrm{wt} \%$, OD 8-15 nm, length 10-50 $\mu \mathrm{m}$ ) and SWNTs (carboxylation $2.73 \mathrm{wt} \%$, OD 1-4 $\mathrm{nm}$, length 5-30 $\mu \mathrm{m}$ ) were purchased from cheaptubes.com. MES hemisodium salt dry powder pH 6.1, $N$-(3-Dimethylaminopropyl)- $N$ 'ethylcarbodiimide hydrochloride (EDC), glycerol phosphate (GP) disodium salt hydrate 
(isomeric mixture), low viscosity chitosan from crab (CS), tris(hydroxymethyl)amino-methane $\left(\left(\mathrm{HOCH}_{2}\right)_{3} \mathrm{CNH}_{2}\right)$ and QuantiPro ${ }^{\mathrm{TM}}$ Bicinchoninic acid (BCA) Assay Kit were purchased from Sigma-Aldrich, UK. L(+)-lactic acid (90\% v/v) and $N$-hydroxysuccinimide (NHS) were obtained from Acros organics, New Jersey, USA. Dialysis membrane (MWCO 12-14 KDa) was obtained from Medicell International Ltd. All salts and acids were obtained from Fisher Scientific (UK). Nylon filter membrane $(0.22 \mu \mathrm{m})$ was purchased from Millipore, UK. Troclosene sodium dihydrate was obtained from Guest Medical (UK).

\subsection{Synthesis of chitosan grafted carbon nanotubes}

Chitosan was covalently grafted on the surface of carboxylated SWNTs and MWNTs according to the method described by Yadav et al. ${ }^{11 \S}$. Grafted CNTs were analysed by ATR and TGA. ATR spectra were obtained with a Varian 640-IR spectrophotometer (Palo Alto, CA, USA). TGA measurements were carried out with a TGA Q50 from TA Instruments, in the temperature range between room temperature and $600^{\circ} \mathrm{C}$, with a heating rate of $10^{\circ} \mathrm{C} / \mathrm{min}$. The degree of grafting was calculated using Eq. 1.

$$
\frac{(\text { chitosan total mass loss } \%) \times x}{(\text { CNTs total mass loss } \%) \times(1-x)}=(\text { grafted CNTs total mass loss } \%) \quad \text { Eq. } 1
$$

Where $x$ is the weight percent of chitosan in the grafted material.

\subsection{Synthesis of HA and HACS composites}

HA and HA composites have been synthesised by wet precipitation method. Briefly, $\mathrm{CaCl}_{2}(12.7$ $\mathrm{ml}, 53 \% \mathrm{w} / \mathrm{v})$ and $\mathrm{NaH}_{2} \mathrm{PO}_{4}(13.3 \mathrm{ml}, 32.5 \% \mathrm{w} / \mathrm{v}), \mathrm{Ca} / \mathrm{P}$ ratio $1: 1.67$, were slowly added to 100 $\mathrm{ml}$ of medium composed of either $\mathrm{HCl} 1.5 \% \mathrm{v} / \mathrm{v}$ or chitosan solution (1\% w/v) in $\mathrm{HCl} 1.5 \% \mathrm{v} / \mathrm{v}$. The $\mathrm{pH}$ was adjusted to 7.0 by slow controlled addition of $\mathrm{NH}_{4} \mathrm{OH}(25 \% \mathrm{w} / \mathrm{v})$. The reaction mixture was magnetically stirred at $37^{\circ} \mathrm{C}$ for 7 days, the $\mathrm{pH}$ was daily checked and adjusted as required. The precipitate was washed once with deionised water, collected by centrifugation ( 2 
min at $2000 \mathrm{rpm})$ and further purified by dialysis against water and freeze dried (at $\left.-40^{\circ} \mathrm{C}\right)$. The materials obtained were characterised by ATR, XRD and TGA. The crystallinity index of HA was calculated for the infra red spectra according to the method described by Weiner and BarYosef $e t a l .{ }^{12}$ A baseline from 750 to $500 \mathrm{~cm}^{-1}$ was drawn and the heights of the two in-plane symmetrical bending bands $v_{4 a}$ and $v_{4 c}\left(600\right.$ and $561 \mathrm{~cm}^{-1}$, respectively) were measured. The $(\mathrm{CI})$ FTIR index was then calculated using Eq. 2:

$$
(\mathbf{C I})_{\text {FTIR-heights }}=\frac{\mathbf{A}(\mathrm{v} 4 \mathrm{a})+\mathbf{A}(\mathrm{v} 4 \mathrm{c})}{\mathbf{A}(\text { (highest point })} \quad \text { Eq. } 2
$$

where $\mathrm{A}(\mathrm{x})$ is the absorbance at the given wavelength and where $\mathrm{A}_{\text {(highest point) }}$ is the signal at the highest point between $v_{4 a}$ and $v_{4 c}$ peaks. XRD analysis was performed with a Rigaku MiniflexII desktop X-ray diffractometer (Tokyo, Japan) with Haskris cooling unit (Grove Village, IL, USA). The tube output voltage used was $30 \mathrm{kV}$, and tube output current was $15 \mathrm{~mA}$. A Cu-tube with Ni-filter suppressing K $\beta$ radiation was used. Measurements were taken from 5 to 40 on the 2 theta scale at a step size of $0.05^{\circ}$ per second in each case. Scans were performed at room temperature.

\subsection{Hydrogels preparation}

Clear solutions of chitosan $(200 \mathrm{mg})$ in lactic acid $(9 \mathrm{ml}, 0.1 \mathrm{M})$ were obtained by overnight stirring at room temperature. Once chitosan was dissolved, samples were sonicated for $30 \mathrm{~min}$ to remove any bubbles and then mixed with glycerol phosphate $(1 \mathrm{ml}, 1.12 \mathrm{~g} / \mathrm{ml})$ for $10 \mathrm{~min}$ at room temperature. The mixtures were then incubated at $37^{\circ} \mathrm{C}$ until gel formation. The sol/gel transition time was determined by the inversion method ${ }^{10}$. When CNTs were included in the composite hydrogels, $15.6 \mathrm{mg}$ of CS-MWNTs or $10 \mathrm{mg}$ of CS-SWNTs $(1.25 \% \mathrm{CNTs})$ were dispersed in lactic acid by sonication for $10 \mathrm{~min}$, before the addition of chitosan. Inclusion of HA and HACS 
was performed by adding $83 \mathrm{mg}$ of either material after dissolution of the chitosan and aiding homogeneous dispersion by sonication for $30 \mathrm{~min}$. Swollen hydrogels were characterised as described below, immediately after formation. Freeze-dried (at $-20^{\circ} \mathrm{C}$ ) hydrogels were prepared by transferring the mixtures into a 24 well plate before gelation and were characterised by ATR, SEM and DVS (see below). SEM images were obtained for gold coated samples (sputter coater Polaron e500, Quoram technologies) with a Jeol JSM-6160L scanning electron microscope with electron backscatter and EDX capability, Silicon Drift Detector (SDD) - X-MaxN, Oxford Instruments.

\subsection{Characterisation of the freeze-dried hydrogels by DVS}

Dynamic vapour sorption (DVS) analysis of the freeze-dried hydrogels was carried out with a Surface Measurement Systems DVS Advantage instrument, using nitrogen as the carrier gas. The mass change of samples subjected to a changing water vapour partial pressure at $25^{\circ} \mathrm{C}$ was recorded. The partial pressure was increased from 0 to $90 \%$ in $10 \%$ increments; the partial pressure was increased to the next step either after equilibrium or after a maximum time of 360 min. A full adsorption/desorption cycle was performed; the data collected were used to calculate the adsorption and desorption isotherms as well as the hysteresis. The data were further analysed according to the following Eq. 3.

$$
w_{p}=K_{p} t^{n_{p}} \quad \text { Eq. } 3
$$

Where $w_{p}$ is the weight gain; $K_{p}$ is the kinetic constant of water penetration into the composite material; $n_{p}$ is the exponent describing the mechanism of water penetration.

2.6. Characterisation of the swollen hydrogels

A TA.XT plus texture analyser (Stable Micro Systems) was used to characterise the hydrogels. Syringeability tests were performed on the composite mixtures before gelation took place. The 
liquid formulations were loaded onto $5 \mathrm{ml}$ plastic syringes fitted with $19 \mathrm{G}, 25 \mathrm{~mm}$ long needles. The syringe was then vertically clamped to a syringe rig and the instrument probe $(10 \mathrm{~mm}$ diameter) was lowered until in contact with the syringe plunger. The probe was used to compress the barrel of the syringe at a constant speed $(1 \mathrm{~mm} / \mathrm{s}$, corresponding to $3.8 \mathrm{ml} / \mathrm{min}$, comparable to normal injection rates) to a distance of $40 \mathrm{~mm}$ and the initial glide force, dynamic glide force and maximum force were measured ${ }^{13}$. All measurements were performed in triplicate at room temperature. Texture profile analysis was then performed by depressing a polycarbonate probe (10 mm dia) into the gels at $1 \mathrm{~mm} / \mathrm{s}$ and to a depth of $5 \mathrm{~mm}$, six measurements were taken at room temperature before and after the sol/gel transition took place. Force/distance curves were obtained and used to calculate the following parameters: 1) Compressibility $(\mathrm{N} \cdot \mathrm{mm})$; work required to deform the product during the compression phase. 2) Adhesiveness $(\mathrm{N} \cdot \mathrm{mm})$; work necessary to overcome the attractive forces between the sample and the probe. Gels prepared by pouring their liquid form into Teflon lined test tubes and incubated at $37^{\circ} \mathrm{C}$ until complete gelation to obtain cylindrical gels $(\mathrm{h}, 10 \mathrm{~mm}$; dia, $12 \mathrm{~mm}$ ) were further tested in compressive mode. Individual samples were placed on a flat plastic surface and compressed at a rate of $1 \mathrm{~mm}$ / $\mathrm{s}$ to a distance of $5 \mathrm{~mm}$, using a stainless compression probe (dia. $40 \mathrm{~mm}$ ). Stress $v s$. strain curves where obtained where the stress $(\mathrm{Pa})$ was expressed as the measured force $(\mathrm{N}) /$ contact area of the sample $\left(1.3 \times 10^{-3} \mathrm{~m}^{2}\right)$ and the strain (no unit) was calculated as distance travelled by the probe $(\mathrm{mm}) /$ height of the sample $(\mathrm{mm})$. The initial Young's modulus of compression was calculated as the slope of the linear portion of the stress-strain curve in the strain range 0.010.05 .

\subsection{In vitro calcification assay}


The bone bonding activity of the composite materials has been assessed in vitro by soaking the materials in standard simulated body fluid (SBF) according to Kokubo's protocol ${ }^{14}$. Freeze-dried hydrogels, prepared in duplicate were immersed in $15 \mathrm{ml} \mathrm{SBF}$ and incubated at $36.5 \pm 2{ }^{\circ} \mathrm{C}$ for 7 and 14 days. SBF was replaced every 4 days. At the final time point, samples were retrieved, washed lightly with purified water and air-dried for few hours before further drying under vacuum. Controls were prepared by soaking in purified water for few hours and drying as described above. All samples were analysed by SEM.

\subsection{BSA loading and release studies}

Hydrogel samples of a total volume of $5 \mathrm{ml}$ were prepared in $15 \mathrm{ml}$ tubes as described above. Before addition of the cross-linker, $5 \mathrm{mg}$ of BSA were dissolved in each sample. The mixtures were then incubated at $37^{\circ} \mathrm{C}$ until gel formation, at this point $5 \mathrm{ml}$ of PBS were added to each sample and samples of $2 \mathrm{ml}$ were taken at predetermined time points. The PBS was replaced with fresh buffer. The concentration of BSA was determined by BCA assay according to manufacturer's instructions. To minimise and take into consideration any possible interference of chitosan with the assay, all samples were centrifuged before analysis and samples containing no BSA were used as controls.

3. Results and discussion

\subsection{Characterisation of the HA materials}

The hydroxyapatite obtained by wet precipitation in presence or absence of chitosan was characterised by XRD, TGA and FTIR (Figure 1). IR spectra (Fig. 1A) of HA and HACS showed the characteristic bands of $\mathrm{PO}_{4}{ }^{2-}$ at 1076-1024 ( $v_{3}$, asymmetric stretching), $962\left(v_{1}\right.$, symmetric stretching), 600 and $561 \mathrm{~cm}^{-1}\left(\mathrm{v}_{4 \mathrm{a}}\right.$ and $\mathrm{v}_{4 \mathrm{c}}$ in-plane bending of the O-P-O, 
respectively $)^{15}$. IR spectrum of the HACS composite showed presence of the characteristic bands of both CS and HA.
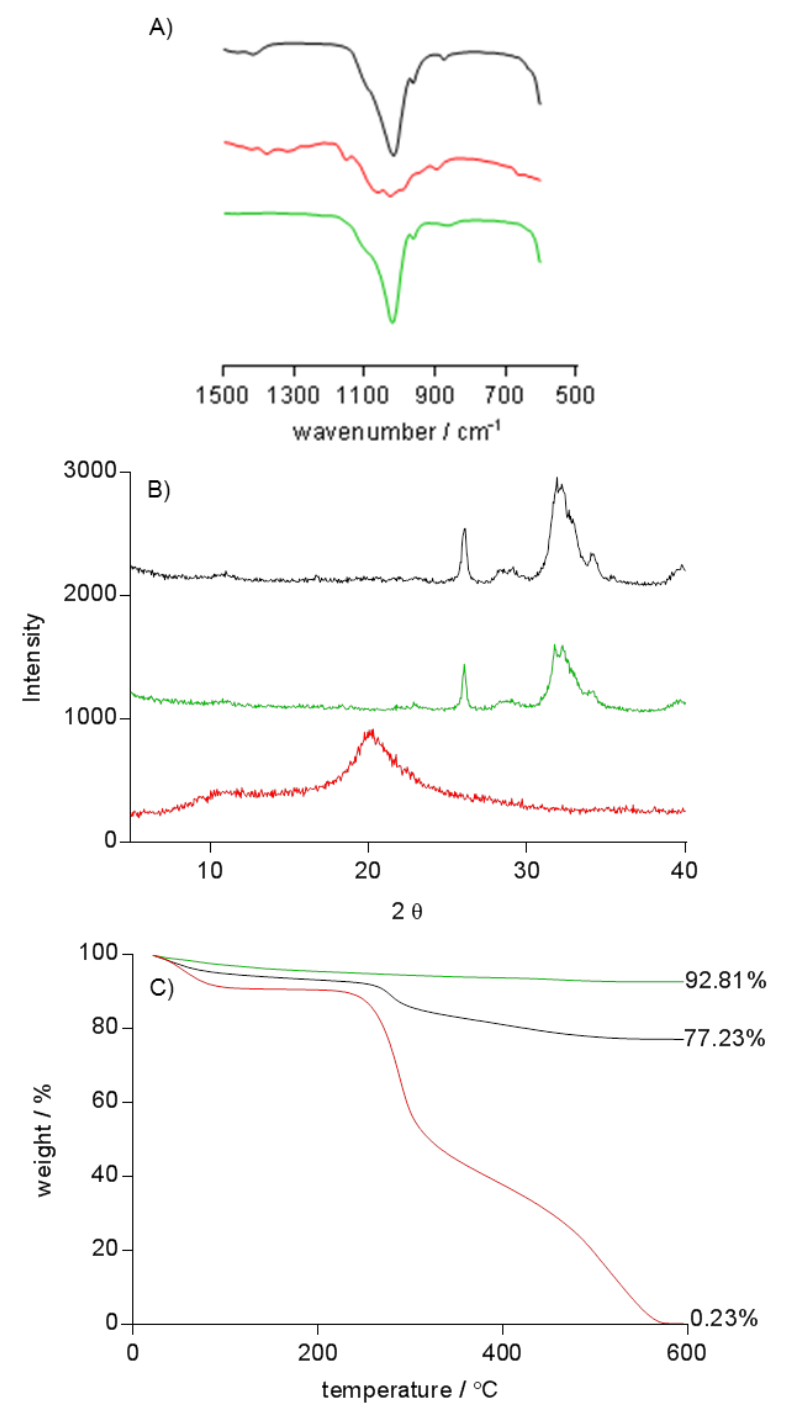

Figure 1. A) FTIR spectra, B) XRD diffractograms and C) TGA profiles of chitosan (red), HA (green) and HACS (black).

The disappearance of the band at $644 \mathrm{~cm}^{-1}(\mathrm{OH})$ and the shift of the CS signals from 1375 to $1413 \mathrm{~cm}^{-1}$ suggested that HA hydroxyl groups might interact with the hydroxyl groups of chitosan via the formation of hydrogen bonds ${ }^{16,17}$. Also the characteristic amino group band of 
the polysaccharide, at $1650 \mathrm{~cm}^{-1}$, almost disappeared while the amide I band at $1589 \mathrm{~cm}^{-1} \mathrm{shifted}$ to $1633 \mathrm{~cm}^{-1}$ and became less intense, indicating electrostatic interactions and hydrogen bonding is probably taking place between the $\mathrm{PO}_{4}{ }^{3-}$ groups of $\mathrm{HA}$ and the $\mathrm{NH}_{3}{ }^{+}$groups of chitosan ${ }^{18}$. From the IR spectra it is possible to calculate the Weiner and Bar-Yosef's Crystallinity Index $\left(\mathrm{CI}_{\mathrm{FTIR}-\text { heights }}\right)$, this is related to the molecular and atomic bonds within the crystal as opposed to the CI obtained by XRD data that is related to the structural arrangement and the size of the crystal $^{12}$. The CI index obtained from the IR data showed a higher value for the composite compared to the HA alone, further confirming the core role of the polysaccharide in the structure of the composite (Table 1).

Table 1. Crystallinity index values obtained from IR and XRD data and crystallite size obtained from XRD data.

\begin{tabular}{llll}
\hline & HA & HACS & Fresh bone \\
\hline CI $_{\text {FTIR-heights }}$ & 3.19 & 4.49 & 2.28 \\
CI $_{\text {XRD / \% }}$ & 0.27 & 0.08 & - \\
Crystallite size / nm & 23.85 & 16.41 & - \\
\hline
\end{tabular}

On the other hand the XRD derived CI indicated that a lower percentage of the material was in a crystalline form when chitosan was included in the composite. The XRD diffractograms (Fig. 1B) showed peaks at $2 \theta=26.05^{\circ}, 32.2^{\circ}, 34.1^{\circ}$ and $39.8^{\circ}$ both in HA and HACS materials; these matched JCPDS card No. 09-432 for $\mathrm{HA}^{19}$. The diffraction patterns observed corresponded to the hexagonal phase of HA and was similar to the apatite in natural bone. Elhendawi et al. ${ }^{16}$ suggested that the CS peaks would not be seen because of the difference in the intensity of the diffraction peaks between HA and CS. TGA results also supported this observation as they indicated that chitosan accounted only for $17 \%$ of the composite weight (Fig. 1C). Chitosan 
degraded completely when heated to $600^{\circ} \mathrm{C}$, after a first water loss at low temperature, it underwent a 2 step degradation process involving depolymerisation, degradation of pyranose rings through dehydration and deamination and lastly ring-opening reactions. ${ }^{1}$ Error! Bookmark not defined. The residue mass observed at $600^{\circ} \mathrm{C}$ was assigned to the presence of inorganic material. The poorly crystalline apatite structure obtained is expected to be more bioactive than a fully developed crystalline hydroxyapatite ${ }^{20}$.

\subsection{Characterisation of the grafted nanotubes}

Homogeneous aqueous dispersions of CNTs are difficult to achieve as they form bundles thermodynamically stabilised by numerous $\pi-\pi$ interactions. Techniques such as ultrasonication and chemical modification of the CNT surface have been used to perturb the extended delocalised $\pi$ system enabling better dispersion of CNTs in aqueous media ${ }^{21,22}$. In the present study chitosan was covalently grafted to carboxylated CNTs to afford stable CNTs aqueous dispersions and to guarantee efficient inclusion of the nanomaterial in the hydrogels, preventing CNTs leaching out of the site of application ${ }^{23}$. A simple coupling procedure involving the use of carbodiimide was used, as this could be carried out at room temperature and in aqueous media, preserving the molecular structures of both chitosan and CNTs (zero-linker) and using nonhazardous reagents. 

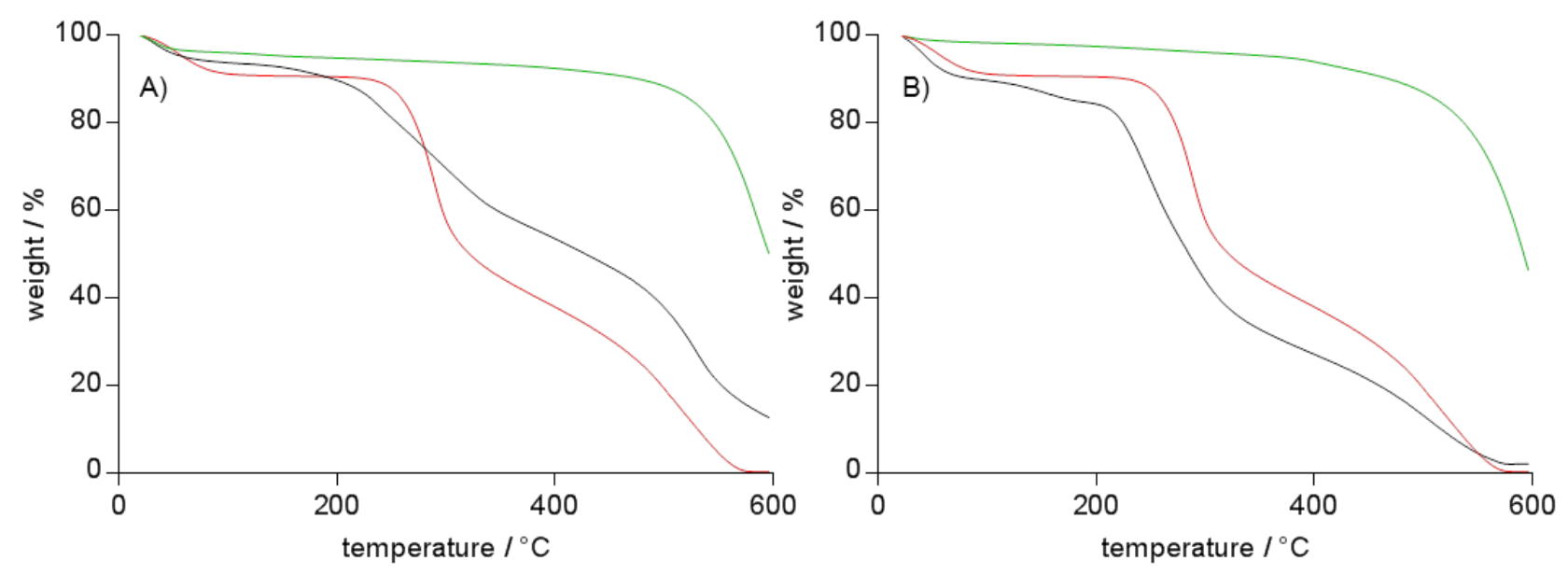

Figure 2. TGA profiles of chitosan (red), CNTs (green) and CS-CNTs (black) where CNTs are SWNTs in A) and MWNTs in B).

The chemical modification of the composites was confirmed by IR (Fig S1 ${ }^{\S}$ ) and TGA (Fig 2). When chitosan was grafted onto CNTs, the peak at $1648 \mathrm{~cm}^{-1}$ of the secondary amide bond disappeared and a noticeable peak near $1633 \mathrm{~cm}^{-1}$ appeared in the spectrum of CS-CNTs, indicating the formation of a new amide bond. The successful amidation reaction for both CSCNTs was also confirmed by the appearance of new peaks between 550 and $1680 \mathrm{~cm}^{-1}$; our results correlate well with previous studies ${ }^{24}$. TGA showed that SWNTs had a higher degree of modification with $84 \%$ of their total weight formed by chitosan as compared to $75 \%$ in CSMWNTs (Fig. 2).

\subsection{Characterisation of the swollen gels}

Table 2. Syringeability data for suspensions containing the composite materials. Data are presented as mean $\pm \mathrm{SD}(\mathrm{n}=3)$. Anova returned a $\mathrm{p}$ value $<0.0001$ for all three parameters. According to Sidak's multicomparison test, all samples were significantly different from water 
$(\mathrm{p}<0.0001)$; samples significantly different from the chitosan solution control (CS) are indicated with $* \mathrm{p}<0.05, * * \mathrm{p}<0.01, * * * \mathrm{p}<0.001$ and $* * * * \mathrm{p}<0.0001$

\begin{tabular}{|c|c|c|c|}
\hline Material & $\begin{array}{l}\text { Initial glide force } \\
(\mathrm{N})\end{array}$ & $\begin{array}{l}\text { Dynamic glide force } \\
\text { (N) }\end{array}$ & $\begin{array}{l}\text { Maximum } \\
(\mathrm{N})\end{array}$ \\
\hline Water & $3.89 \pm 0.82$ & $2.76 \pm 0.25$ & $3.45 \pm 0.47$ \\
\hline CS & $16.11 \pm 1.22$ & $15.30 \pm 0.97$ & $15.52 \pm 0.92$ \\
\hline CS-HA & $17.02 \pm 0.16$ & $15.56 \pm 0.18$ & $15.64 \pm 0.14$ \\
\hline CS-HACS & $19.12 \pm 1.00^{* *}$ & $17.44 \pm 0.78^{* *}$ & $17.57 \pm 0.87^{*}$ \\
\hline CS-HACS-SWNTs & $19.81 \pm 0.81^{* * *}$ & $17.83 \pm 0.79^{* *}$ & $18.17 \pm 0.73^{* *}$ \\
\hline $\begin{array}{l}\text { CS-HACS- } \\
\text { MWNTs }\end{array}$ & $24.31 \pm 0.27^{* * * *}$ & $23.57 \pm 0.31^{* * * *}$ & $24.36 \pm 1.00^{* * * *}$ \\
\hline
\end{tabular}

The evaluation of syringe performance of formulations of human drugs and biologics is an FDA regulatory requirement, this is tested by measuring the force needed to start and maintain the movement of the plunger along the syringe barrel ${ }^{25}$. The composite materials were tested to verify their ease of administration via a syringe. Initial glide force (force required for the initial movement of the plunger), dynamic glide force (force required to maintain the movement of the plunger) and maximum force (highest force measured during the experiment) needed to expel the mixture though a $19 \mathrm{G}$ needle were measured (Table 2). All preparations presented force values below $30 \mathrm{~N}$, considered the upper limit of reasonable injection force as defined by Burkbuchler et al. ${ }^{13}$.

These authors also found that the force needed to inject a liquid into subcutaneous tissue is 1.1 fold higher than that measured in air, indicating that our materials would not go over the $30 \mathrm{~N}$ limit even when injected in vivo. All parameters showed an increase in value when HACS was added, while the addition of HA alone did not have a significant effect on syringeability. This 
might be due to the fact that HA alone does not take part in the gel network and does not affect the flow of the gel subjected to the pressure of the plunger, while the presence of chitosan in the HACS composite allowed for a more intimate contact between HA and the gel structure with participation in the formation of the crosslinking. For this reason the CS-HACS gel provided a higher resistance to flow. Further increase in the syringeability parameters was observed with the addition of both SWNTs and MWNTs, these, being grafted with chitosan, provided further crosslinking points with significant effects on the flowability of the hydrogels. Once ascertained that the composite formulations were injectable, the time to achieve a sol/gel transition at $37^{\circ} \mathrm{C}$ was determined for each formulation.

Table 3. Mechanical characterisation of the composite hydrogels (mean $\pm \mathrm{SD}, \mathrm{n}=6$ ) and their gelation time. One-way ANOVA for compressive stress and Young's modulus, $\mathrm{p}<0.002$ and $\mathrm{p}$ $<0.0001$, respectively.

\begin{tabular}{|c|c|c|c|}
\hline Sample & $\begin{array}{l}\text { Gelation time } \\
\text { (min) }\end{array}$ & $\begin{array}{l}\text { Compressive strength } \\
\text { (Pa) }\end{array}$ & $\begin{array}{l}\text { Young's modulus } \\
\text { (Pa) }\end{array}$ \\
\hline $\mathbf{C S}$ & 60 & $116.3 \pm 18.6$ & $2348.7 \pm 383.7$ \\
\hline CS-HA & 40 & $184.8 \pm 37.6$ & $3178.8 \pm 394.0$ \\
\hline CS-HACS & 13 & $225.8 \pm 13.8^{* *}$ & $3682.2 \pm 365.2^{* *}$ \\
\hline $\begin{array}{l}\text { CS-HACS- } \\
\text { MWNTs }\end{array}$ & 8 & $255.8 \pm 43.4^{* *}$ & $4142.1 \pm 240.3^{* *}$ \\
\hline CS-HACS-SWNTs & 6 & $293.6 \pm 60.1^{* *, \#}$ & $4829.4 \pm 396.8^{* *}$ \\
\hline \multicolumn{4}{|c|}{$\begin{array}{l}{ }^{* *} \mathrm{p}<0.01 \text { compared to chitosan gel; }{ }^{*} \mathrm{p}<0.05 \text { compared to CS-HA; }{ }^{*} \mathrm{p}<0.01 \text { compared to } \\
\text { CS-HA and CS-HACS }\end{array}$} \\
\hline
\end{tabular}


containing CNTs had the most marked decrease in gelation time to 8 and 6 min for MWNTs and SWNTs, respectively.
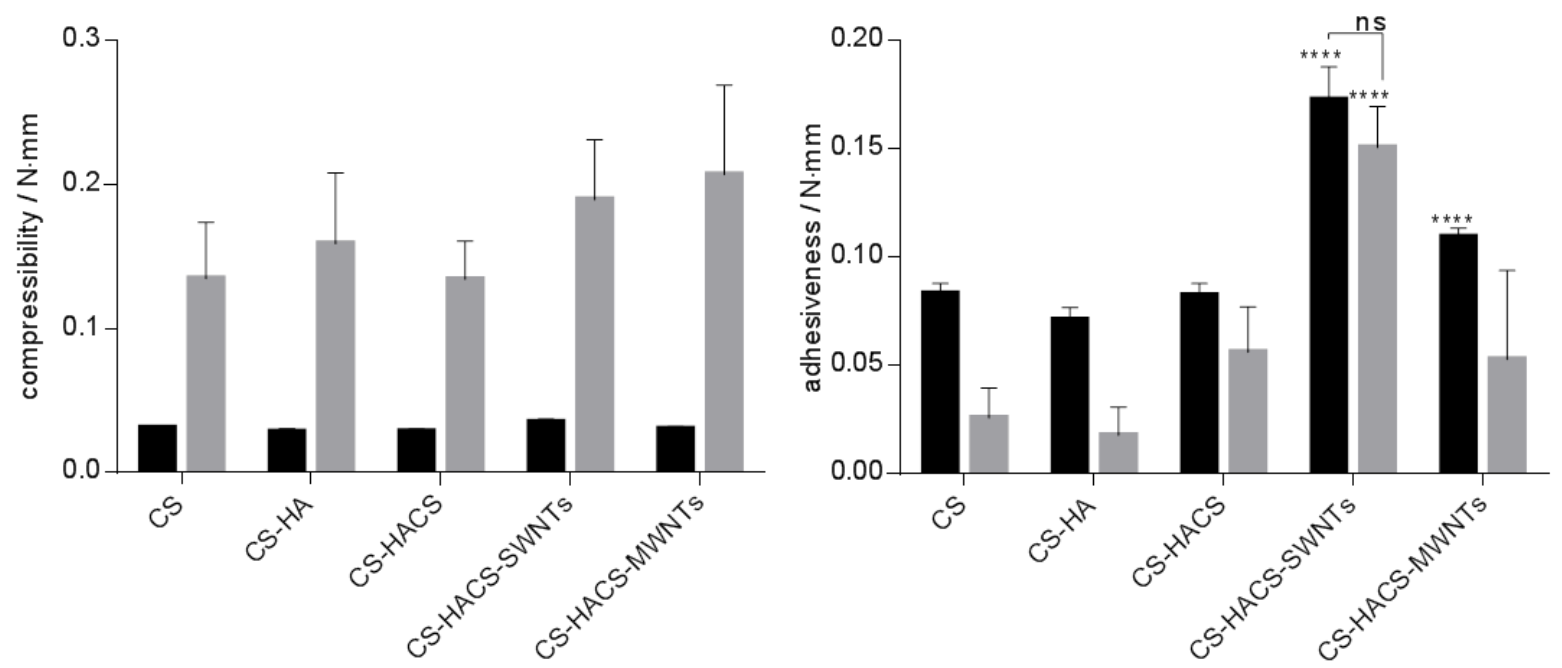

Figure 3. Textural profile of the composite hydrogels. (A) Compressibility, defined as the area under the force vs. distance curve during depression of the probe into the gel; (B) Adhesiveness, defined as the area under the force vs. distance curve during lifting of the probe from the gel. Two-tailed, paired t-test showed that there was a significant difference for all samples and all parameters before and after gelation, except when indicated (ns). One-way Anova was performed and returned $\mathrm{p}<0.05$ for all parameters after gelation. The results of Tukey's multicomparison tests are reported in the graphs $(*, \mathrm{p}<0.05 ; * * * *, \mathrm{p}<0.0001)$. All data are reported as mean \pm $\operatorname{SD}(n=6)$.

This showed that the crosslinking occurring between the three components has a very important role in determining the properties of the composite gels. The gels were further characterised by studying their textural properties ${ }^{26}$. All samples showed a significantly higher compressibility value after gelation confirming that all formulations undergo a sol/gel transition 
at $37^{\circ} \mathrm{C}$ (Fig. 3 and Fig. S2 ${ }^{\S}$ ). Addition of the nanofillers increased, even if not significantly, the compressibility of the materials. Adhesiveness was also significantly changed after gelation took place, with a significant decrease in all cases except for the hydrogel containing SWNTs. This sample also showed a significantly higher adhesion than the chitosan only gel, this fact is interesting as a highly adhesive gel would be more likely to remain on the site of injection for longer. Compression studies carried out on cylinder shaped gels provided more evidence of the role of CNTs in strengthening the gel structures (Table 3 and Fig S3 ${ }^{\S}$ ). The increased Young's modulus and compressive strength observed after addition of CNTs can be attributed to several factors: a higher crosslinking density; high mechanical property of the CNTs reinforcing the matrix; and uniform dispersion of CNTs in the polymer matrix ${ }^{2}$. Comparing the data with information available about the human bone, it is clear that these gels cannot be applied in load bearing joints, however they could be used in non-load-bearing bone or as initial fracture healing callus substitute (Young's modulus $1 \mathrm{MPa}$ ), which can then be remodelled and developed into new bone tissue 27,28 .

\subsection{Characterisation of the freeze-dried gels}

The morphology of the freeze-dried materials was observed by SEM (Fig. 4). The chitosan gel presented a structure with wide pores, the addition of HA and HACS increased the roughness of the surface giving a scaly appearance to the composite with presence of smaller pores together with the wider gaps observed for chitosan alone. Finally, the addition of CNTs afforded composites with a more compact and homogeneous structure but still presenting pores of different sizes. 

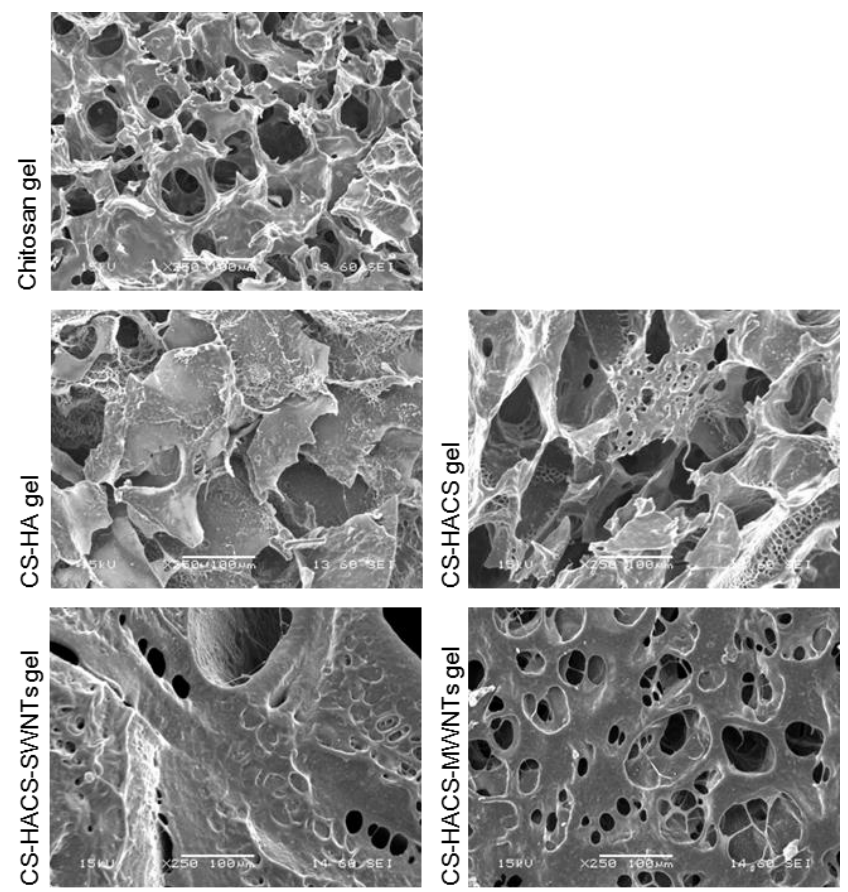

Figure 4. SEM images of freezedried gels.

The gels were further characterised by studying their water sorption and desorption profile at $25^{\circ} \mathrm{C}$ (Fig. 5). A freeze-dried solution of chitosan was used as the control (Fig. 5A). Chitosan showed a profile typical of bulk water sorption, characterised by limited hysteresis and a significant total amount of water absorbed. The hysteresis is due to reversible and elastic swelling deformations as a consequence of water molecules penetration between the chains ${ }^{29}$. Furthermore, chitosan presented a typical sigmoidal shape isotherm (type II of the BDDT classification) indicative of a mechanism of water absorption via formation of a monolayer. The crosslinked chitosan gel presented an increase in the total amount of water absorbed (Fig. 6), as well as in the hysteresis (Fig 5B). Considering that the crosslinking with glycerol phosphate reduced the number of groups available for hydrogen bonding formation with water, the increased absorption can be explained by formation of pores in the structure, as shown by SEM $(\text { Fig. } 4)^{30}$. 

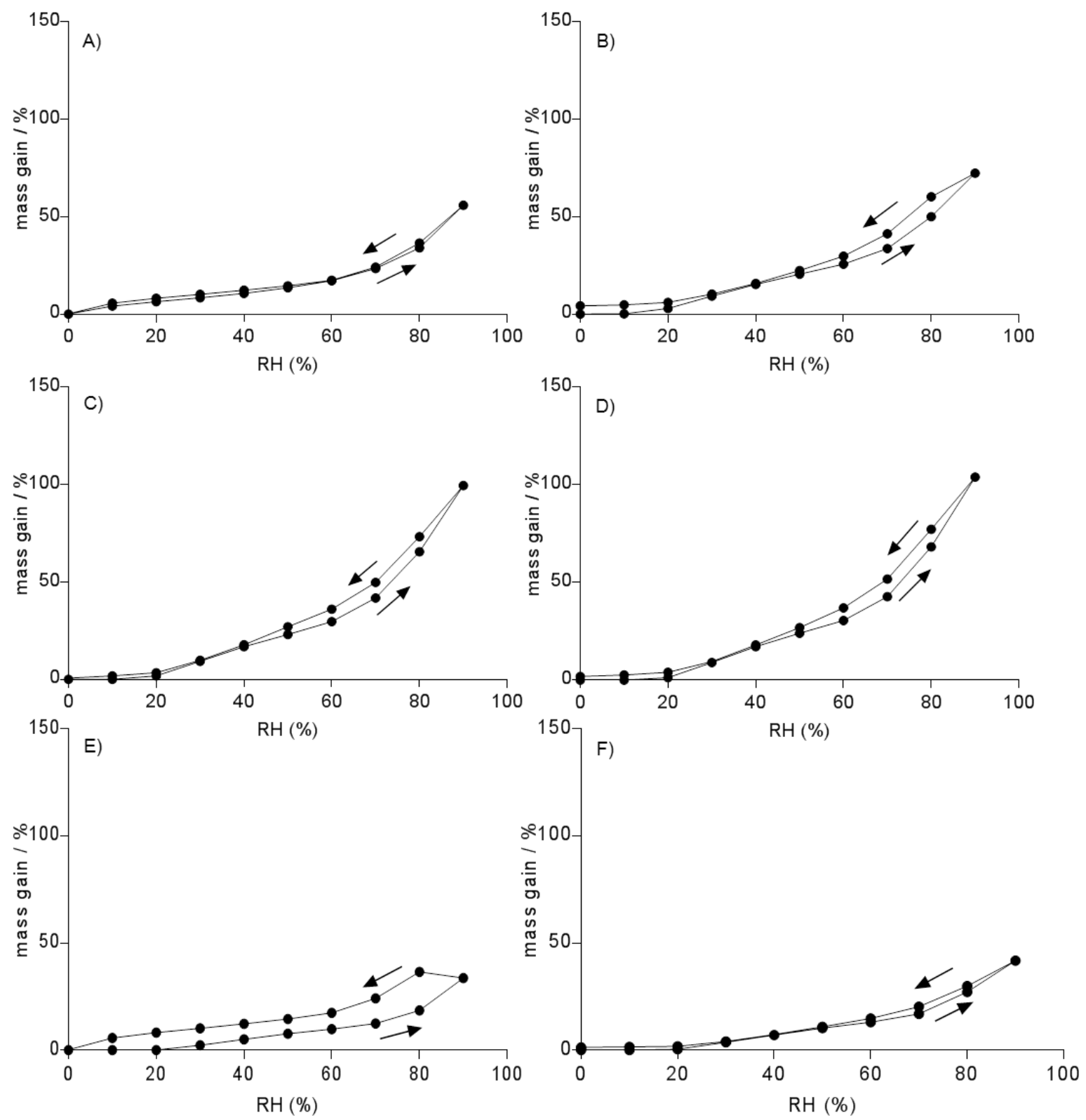

Figure 5. Absorption and desorption isotherms $\left(25^{\circ} \mathrm{C}\right)$ of freeze dried samples: (A) chitosan solution; (B) chitosan gel; (C) chitosan-HA gel; (D) chitosan-HACS gel; (E) chitosan-HACSSWNTs gel and (F) chitosan-HACS-MWNTs gel. 


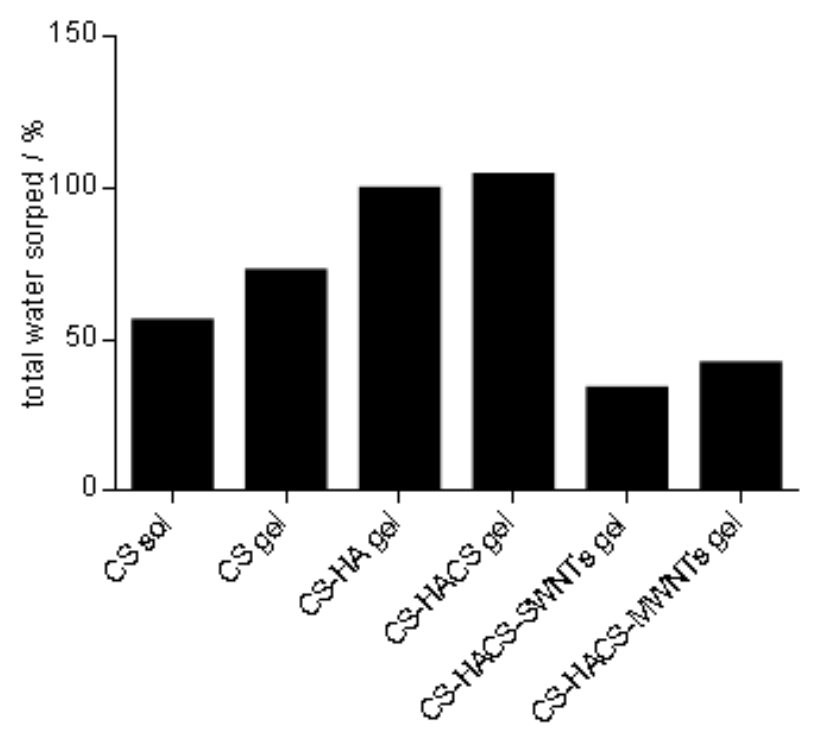

Figure 6. Total amount of water absorbed by the different freeze dried composite materials $(n=1)$.

The isotherm shape also changed to a type III, indicating that a different mechanism of water sorption applies in the case of the gel structure; a cluster mechanism. The addition of HA and HACS resulted in similar effects with increased total amount of water sorbed, type III isotherm and similar hysteresis profile. Furthermore, it was observed that chitosan gel, CS-HA gel and CS-HACS gel, not only were the materials that absorbed the highest amount of water but they all failed to reach equilibrium during the initial drying stage, i.e. after 360 min at RH $0 \%$. This indicates that these materials all contained some bound water, that was not observed in the more hydrophobic, CNTs containing, composites. The inclusion of CNTs in the composite materials also significantly decreased the total amount of water adsorbed, phenomenon that could be explained by the increased hydrophobicity of the material or by the reduction in pore size, as a more compact structure was observed by SEM (Fig. 4). Analysis of the kinetics data (Table 4) revealed that the crosslinked chitosan behaves as a glassy polymer with a case II transport type 
of water penetration kinetic $(n>0.89)$, where the mechanical behaviour of the polymer is the determinant of the penetration kinetic ${ }^{31}$. This behaviour was maintained when HA and CNTs composites where added to the gels with an increased diffusional exponent $(n)$ value possibly due to stiffening of the structure as HA and CNTs are added and an increased importance of the mechanical properties of the material in the determination of the water penetration mechanism.

Table 4. Kinetic parameters values for water penetration in freeze dried hydrogels $(n=1)$.

\begin{tabular}{llll}
\hline Material & $n_{p}$ & $K_{p}\left(\mathbf{m i n}^{-n}\right)$ & $\mathbf{R}^{2}$ \\
\hline CS sol & 0.8890 & 0.0560 & 0.9755 \\
CS gel & 1.6719 & 0.0002 & 0.9904 \\
CS-HA gel & 1.7382 & 0.0001 & 0.9686 \\
CS-HACS gel & 1.5684 & 0.0005 & 0.9502 \\
CS-HACS-SWNTs gel & 1.3205 & 0.0143 & 0.9418 \\
CS-HACS-MWNTs gel & 1.1730 & 0.0017 & 0.9812 \\
\hline
\end{tabular}

These findings were consistent with the fact that all samples exhibited hysteresis implying that the amount of water associated with the solid is greater for the desorption isotherm than the sorption isotherm, at a given relative humidity. This can be explained by changes in polymer chain conformation i.e. chain relaxation or irreversible swelling, molecular ordering, or a combination of these with water penetrating the polymeric structure. The penetration of water into the bulk of the polymer during sorption may be driven by the built-up surface condensed water, but not during desorption, hence resulting in a different mass gain following sorption and desorption at the same relative humidity ${ }^{32}$.

3.5. In vitro calcification of the composite gels 

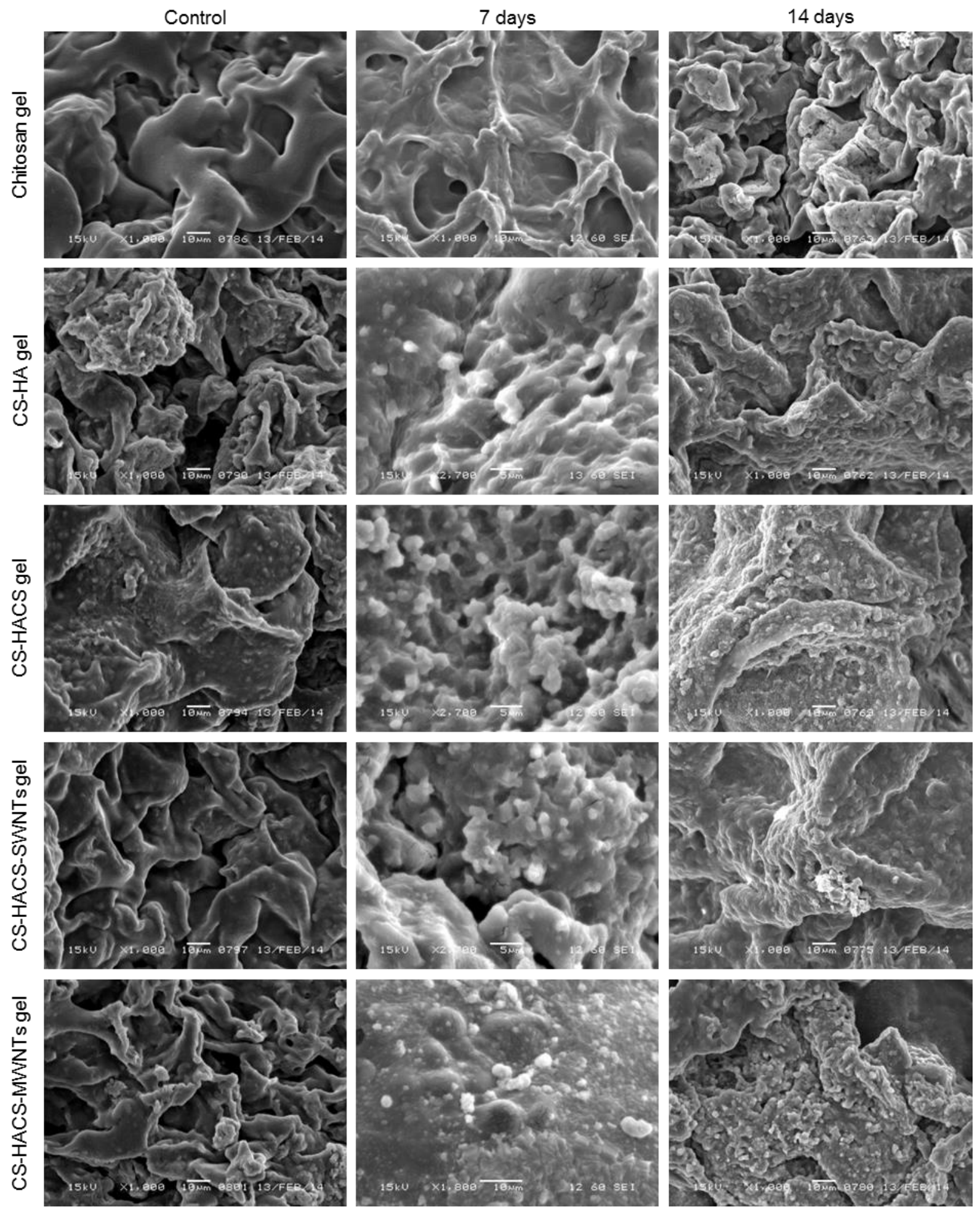

Figure 7. SEM micrographs of hydrogels incubated for 0 (control) 7 and 14 days in SBF at $37^{\circ} \mathrm{C}$. 
In vitro calcification studies were performed to test the bioactivity of the composite materials. SEM was used to visualise the deposition of calcium salts on the surface of the materials after 7 and 14 days of incubation in simulated body fluid. The SEM images (Fig. 7) revealed that cluster deposition of calcium salt started to occur after 7 days in all samples containing HA but not on the chitosan only gel, indicating that HA might be an important nucleating site for deposition of further calcium salts. Additional deposition was observed following incubation for 14 days.

\subsection{Drug release studies}

The release profile of BSA, as a model macromolecule, was evaluated (Fig. 8). The release of the drug was studied from hydrated and formed gels. In clinical application, the gels would be hydrated but administered in the form of viscous suspensions. Since the time of gelation for the 5 formulations studied was significantly different, it would be correct to assume that the gels, that in the in vitro experiment showed very similar release profiles, would show significant differences in vivo, with a marked bust release for those gels that take longer to undergo gelation. All gels, in the conditions used, showed the capacity of controlling drug release over a very long period of time ( $p>0.05$, One-way ANOVA), with a maximum release of about $30 \%$ in 14 days, consistent with similar formulations previously reported ${ }^{33}$. Since the hydrogels are already swollen, it is expected that the mechanism of drug release would follow Fick's law. This was confirmed by the $n$ values obtained by the Korsmeyers Peppas plot (Table 5). All gels presented an $n<0.45$ typical of a case I or simple diffusion mechanism, as opposed to the results obtained for water penetration into the dry gel presented in Table 3 discussed above. 


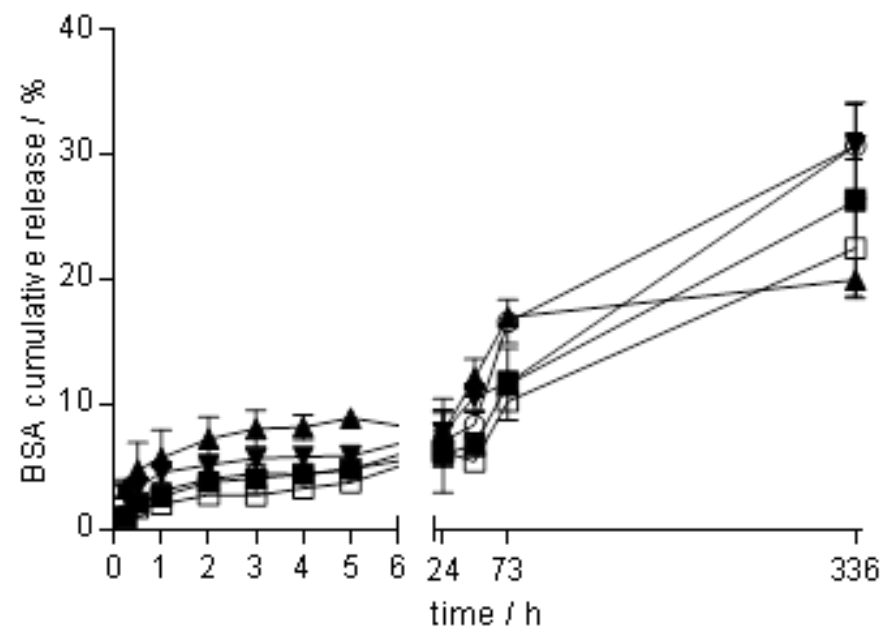

Figure 8. Release of the model protein BSA from gels of the following composition: Chitosan (o

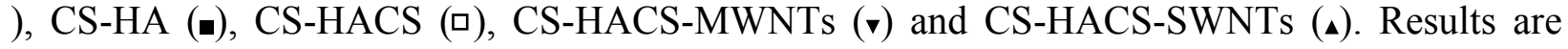
reported as mean $\pm \mathrm{SD}(\mathrm{n}=3)$.

\section{Conclusion}

Thermosensitive injectable composite hydrogels were obtained by crosslinking chitosan with chitosan grafted CNTs and chitosan-HA complexes. The crosslinking strategy allowed for strong and homogeneous interaction between the three materials affording composites with enhanced characteristics. The composite gels underwent rapid sol/gel transition at body temperature and presented enhanced mechanical as well as bioactive properties. The materials developed in the present study are promising scaffolds for bone regeneration.

Associated content

$\S$ Supporting information 
Synthesis of chitosan grafted carbon nanotubes and of HA and HACS composites. ATR spectra of chitosan grafted CNTs, texture profile of composite hydrogels, hydrogels stress strain curves.

\section{AUTHOR INFORMATION}

\section{Corresponding Author}

Marta Roldo, School of Pharmacy and Biomedical Science, University of Portsmouth, St Michael's Building, White Swan Road, Portsmouth, UK. Fax:0044 2392843565; Tel:0044 2392843586; e-mail:marta.roldo@port.ac.uk

\section{Author Contributions}

The manuscript was written through contributions of all authors. All authors have given approval to the final version of the manuscript.

\section{Acknowledgements}

The authors would like to thank Dr Zhongyi Zhang and Dr Loli Serrano López for performing the TGA and XRD experiments, respectively. This research was carried out with the support of the School of Pharmacy and the Faculty of Science, University of Portsmouth, UK.

\section{References}

1. Lienemann, P. S.; Lutolf, M. P.; Ehrbar, M., Biomimetic hydrogels for controlled biomolecule delivery to augment bone regeneration. Advanced Drug Delivery Reviews 2012, 64, (12), 1078-1089.

2. Chen, L.; Hu, J.; Shen, X.; Tong, H., Synthesis and characterization of chitosanmultiwalled carbon nanotubes/hydroxyapatite nanocomposites for bone tissue engineering. Journal of Materials Science: Materials in Medicine 2013, 24, (8), 1843-1851.

3. Cirillo, G.; Hampel, S.; Spizzirri, U. G.; Parisi, O. I.; Picci, N.; Iemma, F., Carbon Nanotubes Hybrid Hydrogels in Drug Delivery: A Perspective Review. BioMed Research International 2014, 2014, 17.

4. Roldo, M.; Fatouros, D. G., Biomedical applications of carbon nanotubes. Annual Reports Section "C" (Physical Chemistry) 2013, 109, 10.

5. $\quad$ Fonseca-Garcia, A.; Mota-Morales, J. D.; Quintero-Ortega, I. A.; Garcia-Carvajal, Z. Y.; Martinez-Lopez, V.; Ruvalcaba, E.; Solis, L.; Ibarra, C.; Gutierrez, M. C.; Terrones, M.; 
Sanchez, I. C.; Del Monte, F.; Velasquillo, M. C.; Luna-Barcenas, G., Effect of doping in carbon nanotubes on the viability of biomimetic chitosan-carbon nanotubes-hydroxyapatite scaffolds. $J$ Biomed Mater Res A 2013.

6. Im, O.; Li, J.; Wang, M.; Zhang, L.; Keidar, M., Biomimetic three-dimensional nanocrystalline hydroxyapatite and magnetically synthesized single-walled carbon nanotube chitosan nanocomposite for bone regeneration Int J Nanomedicine 2012, 7, (1), 2087-2099

7. Lu, X. Y.; Qiu, T.; Wang, X. F.; Zhang, M.; Gao, X. L.; Li, R. X.; Lu, X.; Weng, J., Preparation of foam-like carbon nanotubes/hydroxyapatite composite scaffolds with superparamagnetic properties. Appl Surf Sci 2012, 262, 227-230.

8. Tang, Y.; Zhao, Y.; Li, Y.; Du, Y., A thermosensitive chitosan/poly(vinyl alcohol) hydrogel containing nanoparticles for drug delivery. Polymer Bulletin 2010, 64, (8), 791-804.

9. Gao, C.; Cai, Y.; Kong, X.; Han, G.; Yao, J., Development and characterization of injectable chitosan-based hydrogels containing dexamethasone/rhBMP-2 loaded hydroxyapatite nanoparticles. Materials Letters 2013, 93, 312-315.

10. Nazar, H.; Fatouros, D. G.; van der Merwe, S. M.; Bouropoulos, N.; Avgouropoulos, G.; Tsibouklis, J.; Roldo, M., Thermosensitive hydrogels for nasal drug delivery: The formulation and characterisation of systems based on N-trimethyl chitosan chloride. European Journal of Pharmaceutics and Biopharmaceutics 2011, 77, (2), 225-232.

11. Yadav, S. K.; Mahapatra, S. S.; Yadav, M. K.; Dutta, P. K., Mechanically robust biocomposite films of chitosan grafted carbon nanotubes via the $[2+1]$ cycloaddition of nitrenes. RSC Advances 2013, 3, (45), 23631-23637.

12. Weiner, S.; Bar-Yosef, O., States of preservation of bones from prehistoric sites in the Near East: A survey. Journal of Archaeological Science 1990, 17, (2), 187-196.

13. Burckbuchler, V.; Mekhloufi, G.; Giteau, A. P.; Grossiord, J. L.; Huille, S.; Agnely, F., Rheological and syringeability properties of highly concentrated human polyclonal immunoglobulin solutions. European Journal of Pharmaceutics and Biopharmaceutics 2010, 76, (3), 351-356.

14. Kokubo, T.; Takadama, H., How useful is SBF in predicting in vivo bone bioactivity? Biomaterials 2006, 27, (15), 2907-2915.

15. Koutsopoulos, S., Synthesis and characterization of hydroxyapatite crystals: A review study on the analytical methods. Journal of Biomedical Materials Research 2002, 62, (4), 600612.

16. Elhendawi, H.; Felfel, R. M.; Abd El-Hady, B. M.; Reicha, F. M., Effect of Synthesis Temperature on the Crystallization and Growth of In Situ Prepared Nanohydroxyapatite in Chitosan Matrix. ISRN Biomaterials 2014, 2014, 1-8.

17. Modrzejewska, Z.; Nawrotek, K.; Zarzycki, R.; Douglas, T., Structural Characteristics of Thermosensitive Chitosan Glutaminate Hydrogels. Progress on Chemistry and Application of Chitin and its Derivatives 2013, XVIII, 93-106.

18. Li, X.; Nan, K.; Shi, S.; Chen, H., Preparation and characterization of nanohydroxyapatite/chitosan cross-linking composite membrane intended for tissue engineering. International Journal of Biological Macromolecules 2012, 50, (1), 43-49.

19. Rogina, A.; Ivanković, M.; Ivanković, H., Preparation and characterization of nanohydroxyapatite within chitosan matrix. Materials Science and Engineering: $C$ 2013, 33, (8), 4539-4544. 
20. Kim, H.-M.; Kim, Y.; Park, S.-J.; Rey, C.; HyunMi, L.; Glimcher, M. J.; Seung Ko, J., Thin film of low-crystalline calcium phosphate apatite formed at low temperature. Biomaterials 2000, 21, (11), 1129-1134.

21. Yudianti, R.; Onggo, H.; Sudirman, S. Y.; Iwata, T.; Azuma, J., Analysis of Functional Group Sited on Multi-Wall Carbon Nanotube Surface. The Open Materials Science Journal 2011, 5, (1), 242-247.

22. Fatouros, G. D.; Roldo, M.; Van der Merwe, S. M., Stabilisation of carbon nanotube suspensions. In Carbon Nanotubes: From Bench Chemistry to Promising Biomedical Applications, Pastorin, G., Ed. Pan Stanford Publishing Pte. Ltd.: Singapore, 2011; pp 1-22.

23. Carson, L.; Kelly-Brown, C.; Stewart, M.; Oki, A.; Regisford, G.; Stone, J.; Traisawatwong, P.; Durand-Rougely, C.; Luo, Z., Grafting of Chitosan and Chitosantrimethoxylsilylpropyl Methacrylate on Single Walled Carbon Nanotubes-Synthesis and Characterization. J Chem Chem Eng 2010, 4, (9), 6-13.

24. Wu, Z.; Feng, W.; Feng, Y.; Liu, Q.; Xu, X.; Sekino, T.; Fujii, A.; Ozaki, M., Preparation and characterization of chitosan-grafted multiwalled carbon nanotubes and their electrochemical properties. Carbon 2007, 45, (6), 1212-1218.

25. Cilurzo, F.; Selmin, F.; Minghetti, P.; Adami, M.; Bertoni, E.; Lauria, S.; Montanari, L., Injectability Evaluation: An Open Issue. AAPS Pharmscitech 2011, 12, (2), 604-609.

26. Jones, D. S.; Lawlor, M. S.; Woolfson, A. D., Examination of the flow rheological and textural properties of polymer gels composed of poly(methylvinylether-co-maleic anhydride) and poly(vinylpyrrolidone): Rheological and mathematical interpretation of textural parameters. Journal of Pharmaceutical Sciences 2002, 91, (9), 2090-2101.

27. White, A. A.; Best, S. M.; Kinloch, I. A., Hydroxyapatite-Carbon Nanotube Composites for Biomedical Applications: A Review. International Journal of Applied Ceramic Technology 2007, 4, (1), 1-13.

28. Li, L. H.; Zhao, M. Y.; Ding, S.; Zhou, C. R., Rapid biomimetic mineralization of chitosan scaffolds with a precursor sacrificed method in ethanol/water mixed solution. Express Polymer Letters 2011, 5, (6), 545-554.

29. Bravo-Osuna, I.; Ferrero, C.; Jiménez-Castellanos, M. R., Water sorption-desorption behaviour of methyl methacrylate-starch copolymers: effect of hydrophobic graft and drying method. European Journal of Pharmaceutics and Biopharmaceutics 2005, 59, (3), 537-548.

30. Khodaverdi, E.; Tafaghodi, M.; Ganji, F.; Abnoos, K.; Naghizadeh, H., In Vitro Insulin Release from Thermosensitive Chitosan Hydrogel. AAPS PharmSciTech 2012, 13, (2), 460-466.

31. Ekenseair, A. K.; Peppas, N. A., Network structure and methanol transport dynamics in poly(methyl methacrylate). AIChE Journal 2012, 58, (5), 1600-1609.

32. Agrawal, A. M.; Manek, R. V.; Kolling, W. M.; Neau, S. H., Water distribution studies within microcrystalline cellulose and chitosan using differential scanning calorimetry and dynamic vapor sorption analysis. Journal of Pharmaceutical Sciences 2004, 93, (7), 1766-1779.

33. Kim, G.; Kim, N.; Kim, D.; Kwon, J.; Min, B.-H., An Electrostatically Crosslinked Chitosan Hydrogel as a Drug Carrier. Molecules 2012, 17, (12), 13704-13711. 
TOC graphic

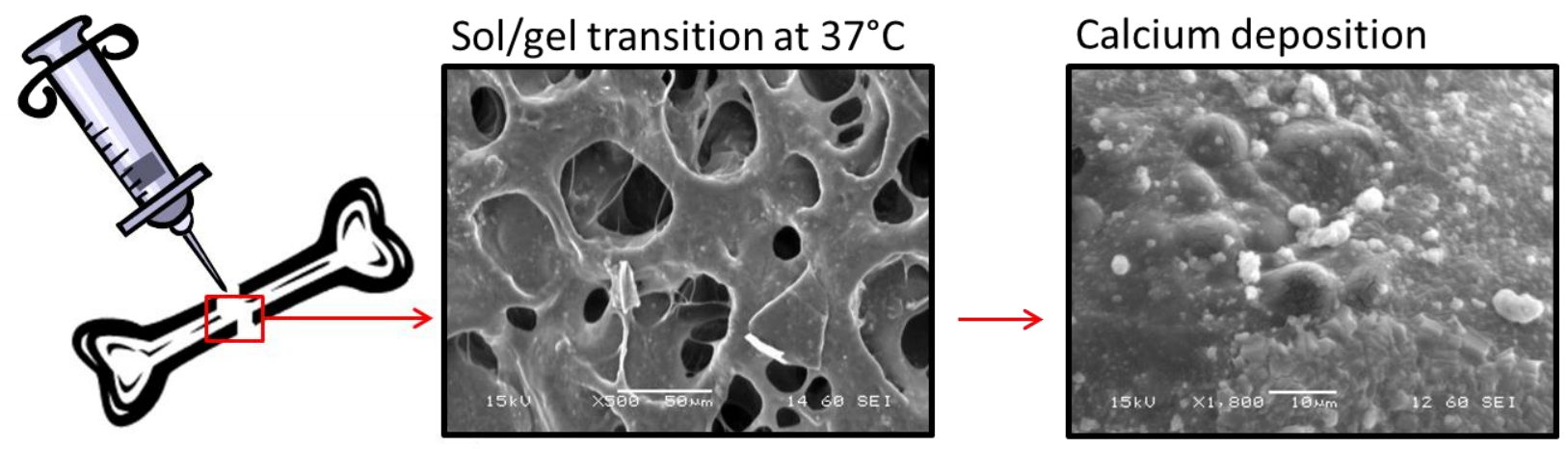

